\title{
THE MIDPOINT SET OF A CANTOR SET
}

\author{
KEN W. LEE \\ Department of Mathematical Sciences \\ Missouri Western State College \\ 4525 Downs Drive \\ Saint Joseph, Missouri 64507 U.S.A.
}

(Received March 28, 1978)

ABSTRACT. A non-endpoint of the Cantor ternary set is any Cantor point which is not an endpoint of one of the remaining closed intervals obtained in the usual construction process of the Cantor ternary set in the unit interval. It is shown that the set of points in the unit interval which are not midway between two distinct Cantor ternary points is precisely the set of Cantor nonendpoints. It is also shown that the generalized Cantor set $C_{\lambda}$, for $1 / 3<\lambda<1$, has void intersection with its set of midpoints obtained from distinct members of $\mathrm{C}_{\lambda}$.

KEY WORDS AND PHRASES. Cantor set, midpoint set, distance set.

AMS (MOS) SUBJECT CLASSIFICATION (1970) CODES. Primary 00 (General), Secondary 04 (Set Theory).

\section{INTRODUCTION.}

J. Rando1ph [6] and N. C. Bose Majumdar [1] have shown that every point in the unit interval is the mean value of a pair of (not necessarily distinct) Cantor ternary points. In 1936, V. Jarnik [5] noted that the set of all Cantor 
points which represent an irrational number has void intersection with its set of distinct midpoints. Here we will characterize all points in the unit interval which are not midway between two distinct Cantor points. We shall also present a class of Cantor-type sets with the property that each member of the class has void intersection with its set of distinct midpoints.

For certain linear sets, it has been shown [1] that there exists a definite relationship between the distance set and the midpoint set of the given set. We will present examples to demonstrate that this relationship cannot be extended to $\mathrm{n}$-dimensional sets as purported in [1].

\section{BASIC CONCEPTS.}

DEFINITIONS. Let $A \subseteq R$. We define the following sets:

$$
\begin{gathered}
M(A)=\{m: x+y=2 m, x, y \in A\} \\
M *(A)=\{m: x+y=2 m, x \neq y, x, y \in A\} \\
D(A)=\{d: d=|x-y|, x, y \in A\}
\end{gathered}
$$

CONSTRUCTION OF THE SET $C_{\lambda}$. Let $0<\lambda<1$. From the closed unit interval delete the open middle segment of length $\lambda$, leaving two closed intervals $I_{11}$ $I_{12}$ each of length $(1-\lambda) / 2$. From each of the intervals $I_{11}$ and $I_{12}$ delete the middle open segment of length $\lambda(1-\lambda) / 2$, leaving four closed, congruent intervals $I_{21}, I_{22}, I_{23}$, and $I_{24}$ of length $(1-\lambda) / 22$. Continue this process inductively. If we let

$$
\begin{aligned}
& A_{1}=I_{11} \cup I_{12} \\
& A_{2}=\underset{\substack{u \\
k=1}}{4} I_{2 k} \\
& A_{n}=\underset{k=1}{u} I_{n k}^{n}
\end{aligned}
$$


then the set $C_{\lambda}$ is defined to be ${ }_{n=1}^{\infty} A_{n}$. Those points in $C_{\lambda}$ which are not endpoints of any $\mathrm{I}_{\mathrm{nk}}$ in the construction of $\mathrm{C}_{\lambda}$ shall be termed non-endpoints of $C_{\lambda}$. We will denote the set of non-endpoints of $C_{1 / 3}$ by $N$.

3. $M *\left(C_{1 / 3}\right)=(0,1)-N$.

In this section we show that the set of points in the unit interval which are not midway between two distinct Cantor ternary points is precisely the set of non-endpoints of $\mathrm{C}_{1 / 3}$.

THEOREM. (B.M. [2]). $M *\left(C_{1 / 3}\right) \geq(0,1)-N$.

We complete the characterization with the following

THEOREM. $N \subseteq(0,1)-M *\left(C_{1 / 3}\right)$.

PROOF. We need only show that if $z \in N$ and $x=y=2 z$ for $x, y \in C_{1 / 3}$, then $x=y=z$. To accomplish this we shall show that the ternary expansion of $2 z$ satisfies the following property:

(*) Every $\mathrm{w} \varepsilon[0,1]$ which can be expressed as

$$
w=. \delta_{1} 1 \delta_{2} 1 \delta_{3} 1 \ldots . \quad(\text { base } 3),
$$

where $\delta_{j}$ is a complex of 0 's if $j$

is odd (or is empty) and $\delta_{j}$ is a complex

of 2 's if $j$ is even (or is empty),

and the digit 1 appears an infinite

number of times, is uniquely expressable

as $w=x+y$, where $x, y \in C_{1 / 3}$ (see [2].).

Since $z \in N$ and if $0<z<1 / 3$, then 


$$
z=\sum_{i=1}^{\infty} 2 \alpha_{i} / 3^{i}
$$

where $\alpha_{1}=0, \alpha_{i} \varepsilon\{0,1\}$ for $i>1$, and the values 0 and 1 are both assumed an infinite number of times, thus, $2 z<2 / 3$ and

$$
2 z=\sum_{i=1}^{\infty} 4 \alpha_{i} / 3^{i}=\sum_{i=1}^{\infty}\left(\alpha_{i} / 3^{i-1}+\alpha_{i} / 3^{i}\right)=\sum_{j=1}^{\infty}\left(\alpha_{j}+\alpha_{j+1}\right) / 3^{j},
$$

where $\left(\alpha_{1}+\alpha_{2}\right) \in\{0,1\}$ and $\left(\alpha_{j}+\alpha_{j+1}\right) \in\{0,1,2\}$ for

$\mathrm{j}>1$. Expressed as a ternary decimal

$$
2 z=\cdot\left(\delta_{1}+\delta_{2}\right)\left(\delta_{2}+\delta_{3}\right)\left(\delta_{3}+\delta_{4}\right) \ldots(\text { base } 3) .
$$

Since $\alpha_{1}=0$ and $\alpha_{j}(j>1)$ assumes only the values 0 or 1 , clearly in the ternary expansion of $2 z$, the digits 0 and 2 can never appear in succession and consequently $2 z$ satisfies $(*)$. Hence if $x+y=2 z, x, y \in C_{1 / 3}$, then since $z \in C_{1 / 3}$, it follows that $x=y=z$.

Now if $2 / 3<z<1$, then $0<1-z<1 / 3$. By symmetry $1-z \in N$. Thus, if $x$ and $y$ are Cantor ternary points such that $x+y=2 z$, then $(1-x)+$ $(1-y)=2(1-z)$ where $1-x, 1-y \in C_{1 / 3}$. It follows from the above argument that $x=y=z$.

4. A PROPERTY OF $M *\left(C_{\lambda}\right), \lambda>1 / 3$.

In this section we demonstrate a class of sets with the property that each member of the class has void intersection with its set of distinct midpoints.

LEMMA. Let $\lambda>1 / 3$ and let $x, m, y \in C_{\lambda}$ be such that $x<m<y$ and $x+y=2 m$. Then $\mathrm{x}, \mathrm{m}$, and $\mathrm{y}$ are always contained in the same closed interval $I_{\mathrm{nk}}$ for each construction stage $n$ of $C_{\lambda}$.

PROOF. We induct on $n$. For $n=1$, it is easily seen that $x, m, y_{\varepsilon} I_{11}$ or $\mathrm{x}, \mathrm{m}, \mathrm{y}_{\varepsilon} \mathrm{I}_{12}$ since $\lambda>1 / 3$. 
Assume that for $n=t, x, m, y \in I_{t p}$ for some $p$. Let $W$ denote the open segment deleted from $I_{t p}$ and 1 et $I_{t+1, j}$ and $I_{t+1, j+1}$ denote the remaining closed intervals obtained from $I_{t p}$ during the $n+1-s t$ construction stage of $C_{\lambda}$. Since $\lambda>1 / 3$, it follows that $|W|>\left|I_{t+1, j}\right|=\left|I_{t+1, j+1}\right|$. It immediately follows that $x, m, y \varepsilon I_{t+1, j}$ or $x, m, y \varepsilon I_{t+1, j+1}$.

THEOREM. For $\lambda>1 / 3, M^{*}\left(C_{\lambda}\right) \cap C_{\lambda}=\emptyset$.

PROOF. Let $x \in C_{\lambda}$ and let $I_{n}(x) \quad(n=1,2, \ldots)$ be the $n$-th stage closed interval in the construction of $C_{\lambda}$ containing $x$. If $x, m, y \varepsilon C_{\lambda}$ are such that $x+y=2 m$ and $x=y$, then it follows that $x=y=m$ by the preceding lemma, since $n_{n=1}^{\infty} I_{n}(x)=\{x\}$. Consequent1y $M *\left(C_{\lambda}\right) \cap C_{\lambda}=\emptyset$.

\section{A NOTE ON MIDPOINT SETS AND DISTANCE SETS}

If $A$ is a symmetric subset of the closed unit interval with $0,1 \varepsilon A$, then it is known [1] that $D(A)=[0,1]$ if, and only if, $M(A)=[0,1]$. Also in [1], the author attempted to generalize this result to higher dimensions with the following statement.

$$
\begin{aligned}
& \text { If } A_{1}, A_{2}, \ldots, A_{n} \text { are symmetric subsets } \\
& \text { of the closed unit interval with } \\
& 0,1 \varepsilon A_{k}(k=1, \ldots, n) \text {, and if } A=A_{1} \times A_{2} \times \ldots \times A_{n} \text {, } \\
& \text { then the distance set of } A, D(A)=\{d: d=|P-Q| \text {, } \\
& P, Q \varepsilon A\} \text { where }|P-Q| \text { denotes the. Euclidean } \\
& \text { distance from } P \text { to } Q \text {, is the interval }[0, \sqrt{n}] \\
& \text { if, and only if, } M\left(A_{k}\right)=[0,1] \quad(k=1,2, \ldots, n) .
\end{aligned}
$$

While it is true that if $M\left(A_{k}\right)=[0,1]$ for each $k$, then $D(A)=[0, \sqrt{n}]$, the converse does not hold.

EXAMPLE 1. If $A_{1}=[0,1]$ and $A_{2}=[0,1 / 4] \cup[3 / 4,1]$, then clearly $D\left(A_{1} \times A_{2}\right)=[0, \sqrt{2}]$, but $M\left(A_{2}\right)=[0,1 / 4] \cup[3 / 8,5 / 8] \cup[3 / 4,1]$.

EXAMPLE 2. It is known (see [3]) that $D\left(C_{\lambda}\right)=[0,1]$ if, and only if, $\lambda \leqq 1 / 3$; consequently $M\left(C_{\lambda}\right)=[0,1]$ if, and only if, $\lambda \leqq 1 / 3$. For $\lambda>1 / 3$, 
$M\left(C_{\lambda}\right) \neq[0,1]$, but it has been shown [4] that $D\left(C_{\lambda} \times C_{\lambda}\right)=[0, \sqrt{2}]$ for $\lambda \leq \sqrt{2}-1$.

\section{REFERENCES}

1. Bose Majumder, N. C. A Study of Certain Properties of the Cantor Set and of an (SD) Set, Bull. Calcutta Math. Soc., 54 (1962), 8-20.

2. Bose Majumder, N. C. On the Distance Set of the Cantor Set - II, Bull. Calcutta Math Soc., 54 (1962), 127-129.

3. Brown, J. and Lee, K. The Distance Set of Certain Cantor Sets, Real Anaylsis Exchange, Vo1. 2, No. 1, 1976, 48-51.

4. Brown, J. and Lee, K. The Distance Set of $\mathrm{C}_{\lambda} \times \mathrm{C}_{\lambda}, \mathrm{J}$. London Math Soc., 15 (1977), 551-556.

5. Jarnik, V. Sur les Fonctions de Deux Variables Reeles, Fund. Math., 27 (1936), 147-150.

6. Randolph, J. Distances Between Points of the Cantor Set, American Math. Monthly, 47 (1940), 549-551. 


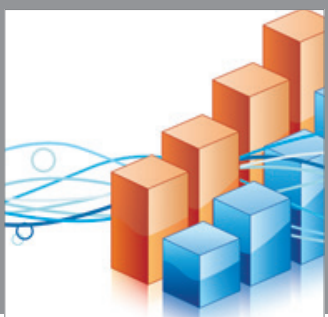

Advances in

Operations Research

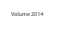

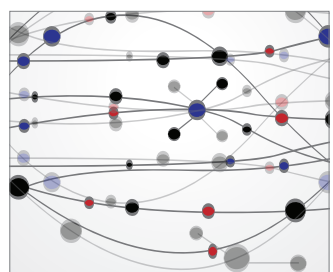

\section{The Scientific} World Journal
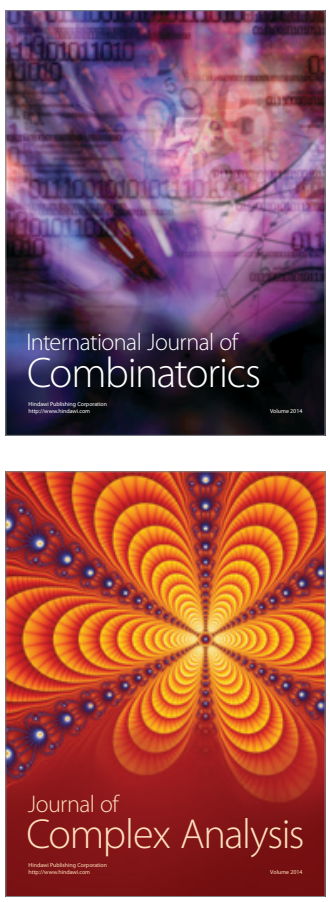

International Journal of

Mathematics and

Mathematical

Sciences
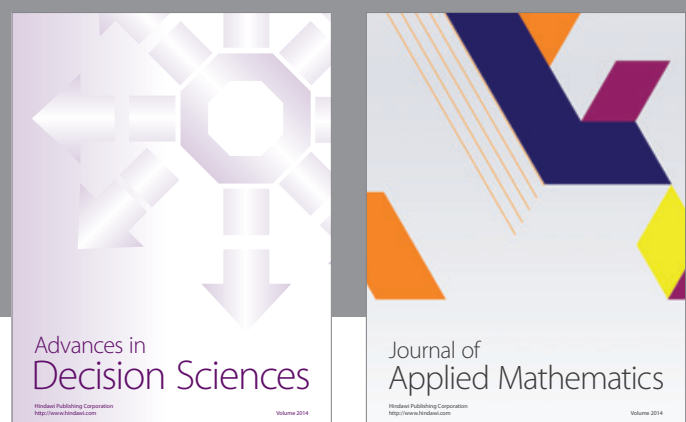

Journal of

Applied Mathematics
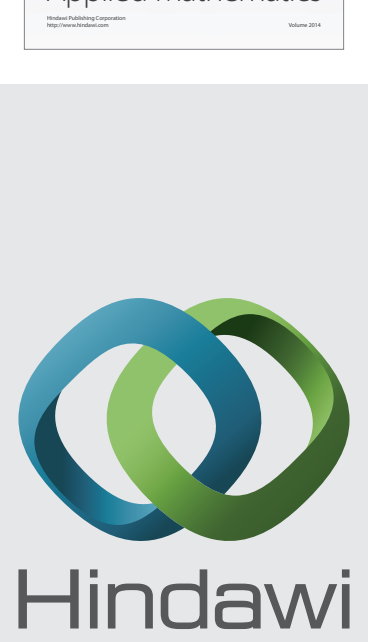

Submit your manuscripts at http://www.hindawi.com
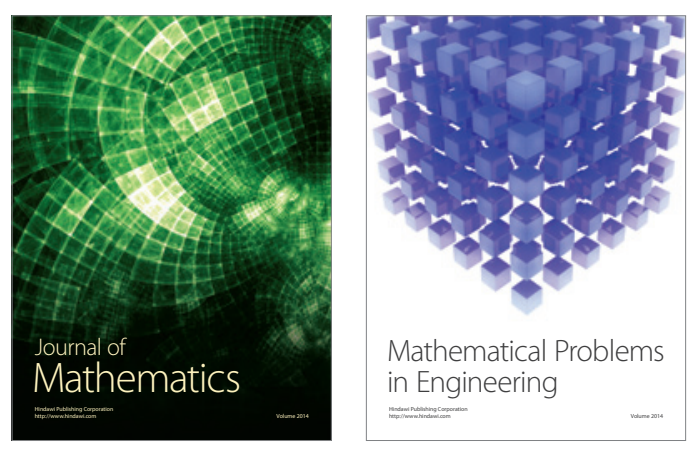

Mathematical Problems in Engineering
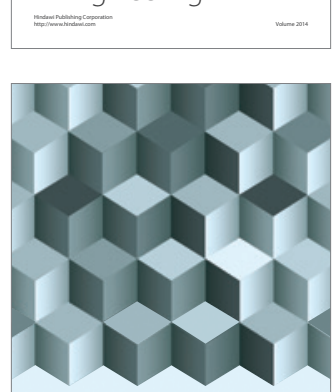

Journal of

Function Spaces
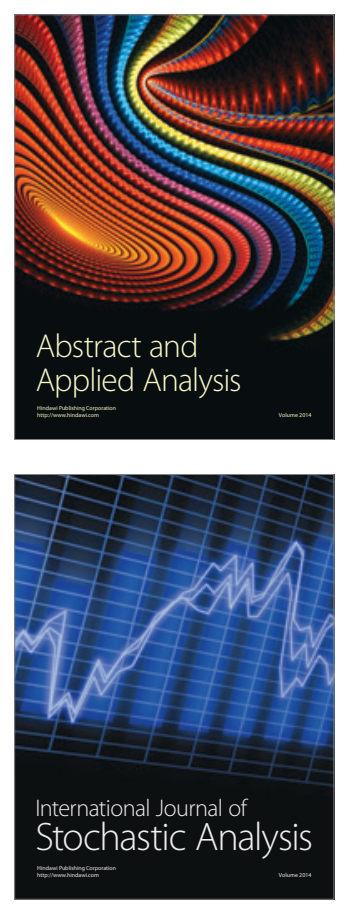

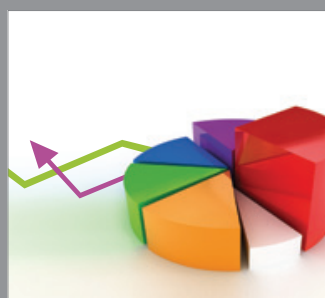

ournal of

Probability and Statistics

Promensencen
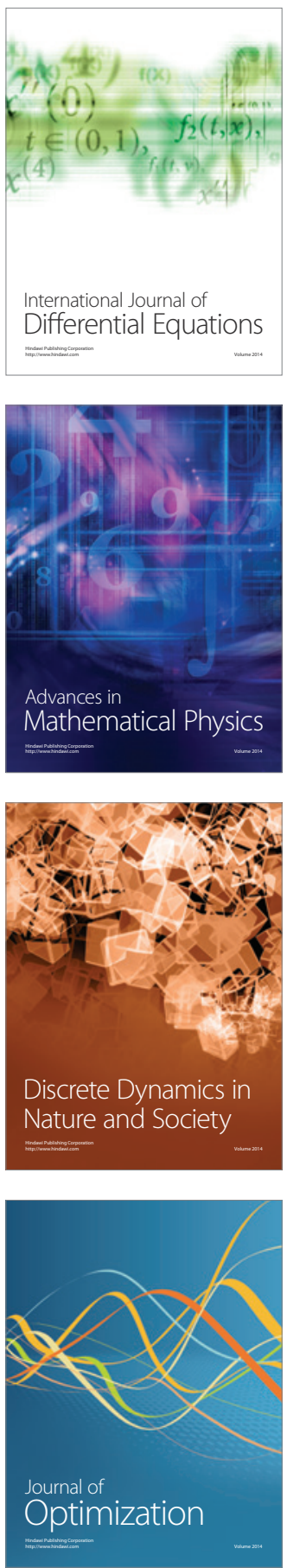